\title{
Perfil de ácidos grasos en leche de vacas Chinampas (Bos taurus) alimentadas con forraje fresco de matorral sarcocaulescente o heno de alfalfa
}

\author{
Fatty acids profile in milk of Chinampo cows (Bos taurus) fed fresh forage \\ from sarcocaulescent shrubs or alfalfa hay \\ R Ortega-Pérez ${ }^{\mathrm{a}, \mathrm{b}}$, JL Espinoza-Villavicencio ${ }^{\mathrm{a}^{*}}$, E Palacios-Mechetnov ${ }^{\mathrm{b}}$, A Palacios-Espinosa ${ }^{\mathrm{a}}$, \\ O Arjona-López ${ }^{b}$, B Murillo-Amador ${ }^{b}$, F Rivera-Acuña ${ }^{c}$ \\ aDepartamento de Zootecnia, Universidad Autónoma de Baja California Sur, La Paz, B.C.S., México. \\ bCentro de Investigaciones Biológicas del Noroeste, La Paz, B.C.S., México. \\ cDepartamento de Medicina Veterinaria y Zootecnia, Instituto Tecnológico de Sonora, Sonora, México.
}

\begin{abstract}
SUMMARY
The objective of the present study was to determine the concentration of fatty acids (FA) in the milk of Chinampo cows (Bos taurus), with emphasis on conjugated fatty acids (CLA). A sample of $10 \mathrm{ml}$ was collected from 32 cows that had been lactating at least 60 days, but not longer than 120 days. A group $(\mathrm{FV}, \mathrm{n}=25$ ) was free-ranged grazed on green fresh pasture (sarcocaulescent shrub semiarid area, La Paz, B.C.S.) during the wet season (SeptemberOctober 2009). The second group (HA, $n=7)$ was penned and fed fodder of alfalfa hay. The FA analysis revealed that there were less saturated FA in the FV group $(\mathrm{P}<0.05)$. This difference was basically explained by the lesser content of 14:0, 15:0, and 16:0. The concentration of total monounsaturated FA was higher in FV $(\mathrm{P}<0.05)$, given by the higher concentration of 18:1 $n-9$ and 18:1 $n-7$ trans-11. The total concentration of polyunsaturated FA was similar for both groups, but there was a higher concentration of 18:2 $n-6$ and 18:2n-7 cis-9 trans-11 in the FV group $(\mathrm{P}<0.01)$. The proportion of omega-3 FA was similar for both groups, but the concentration of omega-6 FA was higher in FV ( $\mathrm{P}<0.01)$, resulting in an increased omega-6:omega-3 ratios in the FV group compared to HA. It is concluded that Chinampo cows left to free-range on fresh green pasture reduced the total saturated FA of medium chain length, and increased the concentration of 18:1 n-9, 18:1 n-7 trans-11 and 18:2 n-7 cis-9 trans-11, a benefit for human health. However, an increase in omega-3 FA is desirable in the milk of free-ranging cows in the area.
\end{abstract}

Palabras clave: vacas, nutrición, ácidos grasos, leche.

Key words: cows, nutrition, fatty acids, milk.

\section{INTRODUCCIÓN}

La grasa de la leche y de productos lácteos contribuye al consumo de ácidos grasos (AG) esenciales, proteínas y vitaminas en la dieta humana y juega un papel crítico en las propiedades sensoriales de esos alimentos (Chilliard y Ferlay 2004). Las guías de nutrición para humanos enfatizan la importancia de mantener una dieta balanceada para ayudar a reducir la incidencia de problemas en la salud como la obesidad, diabetes tipo-2, cáncer y cardiopatías (Jacobs y col 2004). Los AG juegan un papel determinante en estos problemas de salud. En muchos países desarrollados el alto consumo de AG saturados está asociado con la leche y productos lácteos; esto ha contribuido a una imagen negativa de los mismos (Valsta y col 2005). Por ejemplo, se recomienda que para disminuir la incidencia de ciertos problemas de salud el alimento debe tener una relación de AG omega-6:omega-3 lo más baja posible, así como

Aceptado: 12.10.2012.

* Nicolás Bravo \# 419, entre Guillermo Prieto y Serdán, Colonia Centro, CP 23000, La Paz, B.C.S., México; jlvilla@uabcs.mx una proporción baja de AG saturados, que no deberían de ascender a más del 15-30\% (Dewhurst y col 2006).

La grasa de la leche de vaca contiene una gran proporción (70-75\%) de AG saturados (Lock y Shingfield 2004). Los AG saturados de cadena mediana (por ejemplo el 12:0, 14:0 y 16:0), los cuales representan la mayor parte de los AG saturados en la leche, han sido relacionados con un aumento de la concentración de colesterol y de lipoproteínas de baja densidad (Williams 2000). Sin embargo, la cantidad de cada tipo de AG no es suficiente para determinar su efecto fisiológico, hay que considerar también la relación entre AG. Así, Clandinin y col (2000) sugieren que el AG 16:0 puede no tener efectos negativos si se consume una cantidad adecuada de AG 18:2 n-6. Asimismo, se han descrito mayores efectos de la relación omega-3:omega-6, que de la cantidad total de cada tipo de omega que hay en un alimento (Simopoulos 2008).

El ácido 18:1 n-9 es el AG monoinsaturado (MUFA, por sus siglas en inglés) más abundante en la leche, el resto son en su mayoría isómeros trans de 16:1, 18:1 (Destaillats y col 2000) y 20:1 (Cruz-Hernández y col 2004). La grasa de la leche es también una fuente importante de ácido linoleico conjugado (CLA, por sus siglas en inglés). El 
CLA es un término colectivo que describe una mezcla de isómeros dienoicos, geométrica y posicionalmente conjugados derivados del ácido 18:2 n-6. Así, se ha reportado la presencia de 14 isómeros de CLA (Lock y Bauman 2004), pero el mayor interés se ha enfocado al CLA 18:2 n-7 cis-9 trans-11 y CLA 18:2 n-6 trans-10 cis-12 porque tienen función anticancerígena, antiarteriogénica, inmunomoduladora, promueven el crecimiento y una masa corporal magra (Tanaka 2005).

El CLA 18:2 n-7 cis-9 trans-11 es el isómero predominante, representa el $75-90 \%$ del total de CLA en la grasa de la leche de los rumiantes y se conoce con el nombre común de ácido ruménico, debido a su relación única con los animales pertenecientes a dicho suborden (Kramer y col 1998). Mientras que el ácido ruménico está asociado a los efectos benéficos descritos antes, los efectos de otros CLA y el ácido vaccénico (18:1 n-7 trans-11) se ha correlacionado en algunos estudios con la aparición de ciertos tumores (Field y col 2009). Sin embargo, en otros se ha indicado un efecto benéfico del ácido 18:1 n-7 trans-11 por ser el precursor del ácido 18:2 n-7 cis-9 trans-11, y por su propia cuenta, por modular el efecto del PPAR $\alpha$ (receptor activado por el inductor de la proliferación de peroxisomas tipo alfa) que disminuye los niveles de triglicéridos en la sangre (Moya-Camarena y col 1999) y disminuye la acumulación de grasa en particular cuando la dieta es rica de AG saturados. Así, lo ideal sería incrementar el ácido ruménico y el ácido vaccénico en la leche, sin incrementar los otros CLA hasta que se sepa si son benéficos o no para los consumidores.

Aunque hay numerosos estudios sobre los CLA en leche, la dificultad para identificar los ácidos grasos trans, la diversidad de razas, sistemas de alimentación y tipos de dieta, así como la época del año y otros factores, hacen difícil la comparación de AG en leche de vaca en distintos trabajos (Khanal y Olson 2004). Por ejemplo, se ha observado que la raza Holstein produce más CLA que la Jersey (Lawless y col 1999). No se han encontrado diferencias en el CLA en relación al tiempo de lactancia (Kelsey y col 2003), pero sí en relación a la época del año (Månsson 2008). Se han reportado mayores niveles de CLA en leche de vacas alimentadas con pasto fresco que en seco y mayor CLA cuando hay diversidad de plantas forrajeras (Collomb y col 2004).

La adición de forraje verde a la dieta de rumiantes aumenta la proporción de AG insaturados en la leche (Ferlay y col 2008, Frelich y col 2009, Coppa y col 2011) y en la carne (Duckett y col 2009, Leheska y col 2008). El contenido de CLA en particular es hasta cinco veces más abundante (Dhiman y col 1999). Una razón por la cual el forraje verde promueve esos incrementos es que la mayoría de los AG que contiene son 18:2n-6, precursor de los CLA en el rumen, y 18:3n-3 precursor de 18:1 $n-7$ trans-11, este último, intermediario en la síntesis de 18:2 n-7 cis-9 trans-11 en la glándula mamaria (Griinari y
Bauman 1999). El contenido del ácido 18:3n-3 se reduce a la mitad en el heno con respecto al forraje verde (Izumi y col 2002). Por otro lado, el pH del rumen afecta las especies de microorganismos que inducen la biohidrogenación, siendo mayor la producción de CLA a $\mathrm{pH}$ mayores de 6,0 , lo cual sucede con forraje fresco (Martin y Jenkins 2002). Entonces, la alimentación con forraje verde en lugar de materiales conservados o concentrados parece ser un medio simple y efectivo para aumentar el contenido de CLA en la leche de rumiantes (Tanaka 2005).

En las zonas de mayor aridez del estado de Baja California Sur, México, el sistema de producción de bovinos para carne se basa en el pastoreo libre de ganado criollo nativo, explotado en un sistema de doble propósito. Este ganado tiene su origen en los bovinos introducidos a la península de Baja California durante la conquista española. Los animales fueron expuestos al clima local cálido y seco durante muchas generaciones y la selección natural dio lugar a un bovino adaptado, conocido localmente como Chinampo (Espinoza y col 2009). Las vacas se ordeñan durante la temporada de lluvias, cuando la disponibilidad de forraje verde es suficiente para cubrir sus requerimientos nutricionales. La leche que se obtiene se destina al consumo familiar, principalmente. Considerando los planteamientos anteriores, así como las particularidades y la relevancia de los AG de la leche en la salud humana y la influencia de la dieta del bovino sobre la presencia de algunos de ellos, el objetivo de este estudio fue determinar el contenido de AG en la leche de vacas Chinampas, sometidas a distintos sistemas de alimentación.

\section{MATERIAL Y MÉTODOS}

\section{ÁREA DE ESTUDIO Y TRATAMIENTOS}

El trabajo se llevó a cabo en el municipio de La Paz, B.C.S., México, ubicado en las coordenadas geográficas 26²4'16" N y 10954'49" O. Entre septiembre y octubre (estación de lluvias) de 2009 se colectaron muestras de leche de vacas Chinampas que pastorearon forraje verde de manera permanente en un agostadero abierto (FV; $\mathrm{n}=25$ ) y de vacas del mismo grupo racial mantenidas en corral y alimentadas para cubrir sus requerimientos de MS con heno de alfalfa que contenía un $15 \%$ de PC (HA; $n=7$ ). De acuerdo con trabajos previos (Ávalos y col 2009) realizados en la misma área de pastoreo en donde se mantuvo al grupo FV y durante la misma época del año, la disponibilidad de MS es suficiente para cubrir los requerimientos de este tipo de animales, el aporte de PC es ligeramente insuficiente y la EM mayor a la requerida. Las especies vegetales disponibles en el área de pastoreo, a las cuales tuvieron libre acceso los animales del grupo FV, corresponden a un matorral sarcocaulescente y la flora está constituida principalmente por especies arbustivas propias de zonas desérticas como Bursera microphylla, Cyrtocarpa 
edulis, Fouquieria diguetii, Jatropha cinerea, Cercidium floridium, Olneya tesota, Lycium californicum, Merremia aurea, Esenbeckia flava, Haematoxylon brasiletto, Yucca valida, Pithecellobium confine, Krameria parvifolia, Ferocactus peninsulae, Prosopis juliflora, Pachycereus pringlei y algunos pastos de los géneros Bouteloua babata, Aristida sp, Hyptis sp (Morelos 1988).

Las vacas utilizadas fueron animales de dos a cinco partos y al momento del muestreo tenían entre 60 y $120 \mathrm{~d}$ de lactación. El peso vivo y la condición corporal, según Whitman (1975), en las vacas de ambos tratamientos al mismo tiempo fueron de $320 \pm 7,0 \mathrm{~kg} \mathrm{y} 3,0$, respectivamente.

\section{MUESTREOS Y TÉCNICAS DE LABORATORIO}

De cada vaca se tomó una muestra de $10 \mathrm{ml}$ de leche en un tubo estéril; las muestras se colocaron en una hielera para ser transportadas al laboratorio donde se congelaron a $-80{ }^{\circ} \mathrm{C}$ hasta su procesamiento.

La extracción de lípidos se realizó utilizando $2 \mathrm{ml}$ de leche, a los cuales se les agregaron $18 \mathrm{ml}$ de cloroformometanol (1:2) en un vial que contenía butil-hidroxitolueno (BHT) como antioxidante y ácido tricosanoico (23:0) como estándar interno. Los lípidos se extrajeron de acuerdo a la metodología propuesta por Bligh y Dyer (1959). Los lípidos extraídos se conservaron en una atmósfera saturada de nitrógeno hasta su análisis y la cuantificación de lípidos totales se realizó por un método gravimétrico.

Las muestras de leche fueron transesterificadas de acuerdo a la metodología propuesta por Chand y col (2001); se agregó $1 \mathrm{ml}$ de $\mathrm{BF}_{3}-$ Metanol $(10 \%)$ y posteriormente $200 \mu \mathrm{lde} \mathrm{KOH}$ al $1,5 \%$ durante 30 minutos, a $30^{\circ} \mathrm{C}$. La separación de los CLA y ácidos grasos metilesterificados (FAME) se realizó utilizando una columna DB-23 de sílica fundida (50\%-Cianopropil)-metilpolisiloxano) de $60 \mathrm{~m} \times 0.25 \mathrm{~mm}$ ID x $0.25 \mathrm{~mm}$ film (J\&W Scientific) con una rampa de temperatura de $60{ }^{\circ} \mathrm{C}$ a $220^{\circ} \mathrm{C}$ y utilizando un detector de ionización de flama (FID) adaptado en un cromatógrafo de gases (CG) $6890 \mathrm{~N}$ marca Agilent Technologies.

La identificación y cuantificación de los FAME se hicieron con base en estándares externo e interno, respectivamente (Sigma; Bellefonte, PA, USA). Los CLA se identificaron usando (el 18:2 n-7 cis-9 trans-11 y 18:2 n-6 trans-10 cis-12), estándares externos (Nu-Chek Prep, Inc, Elysian, MN, USA) y el 18:1 n-7 trans-11 de Sigma (Bellefonte, PA, USA) y su identidad se verificó usando un $\mathrm{CG}$ conectado a un espectrofotómetro de masas.

\section{ANÁLISIS ESTADÍSTICO}

Para el análisis estadístico se utilizó un modelo lineal general (SAS 2001). Las variables independientes fueron el tipo de alimento consumido por las vacas y las variables de respuesta, los distintos AG medidos en la leche (g/100 g de AG).

\section{RESULTADOS}

La diferencia en la concentración de lípidos totales en la leche de vacas alimentadas con heno de alfalfa (HA; $2,3 \pm 0,67 \mathrm{~g} / 100 \mathrm{~g})$ o que consumieron forraje verde en pastoreo $(\mathrm{FV} ; 3,1 \pm 0,61 \mathrm{~g} / 100 \mathrm{~g})$ fue no significativa $(\mathrm{P}>0,05)$.

\section{ÁCIDOS GRASOS SATURADOS}

El contenido de AG saturados en la leche se presenta en el cuadro 1. El total de AG saturados fue menor $(\mathrm{P}<0,05)$ en las vacas alimentadas con forraje verde comparadas con las vacas alimentadas con heno de alfalfa. Los principales AG saturados que contribuyeron a la diferencia entre los tratamientos fueron los de cadena mediana (14:0, 15:0, 16:0). Los más abundantes fueron 16:0 y 14:0. A diferencia de los AG de cadena mediana, el contenido de 18:0 fue mayor en las vacas del grupo FV. No se observaron diferencias para los AG saturados de cadena larga.

\section{ÁCIDOS GRASOS MONOINSATURADOS (MUFA)}

Los MUFA representaron el 29,4 y 36,8 g por $100 \mathrm{~g}$ de los AG en la leche de las vacas de los grupos HA y FV, respectivamente, $\mathrm{y}$ fueron significativamente diferentes entre sí $(\mathrm{P}<0,05)$. En el cuadro 2 se puede observar que el contenido de algunos MUFA de cadena mediana (14:1 $n-5,16: 1 n$-7 y 17:1 n-7) se redujo en las vacas alimentadas con forraje verde. Sin embargo, en este grupo fue significativamente mayor la concentración de AG de cadena larga como 18:1 n-9, 20:1 n-11, 20:1 n-7 y el 24:1 n-9.

Cuadro 1. Concentración (media $\pm \mathrm{EE}$ ) de ácidos grasos saturados ( $\mathrm{g} / 100 \mathrm{~g}$ de $\mathrm{AG}$ ) en la leche de vacas Chinampas alimentadas con heno de alfalfa (HA) en confinamiento o con forraje verde $(\mathrm{FV})$ en pastoreo.

Concentration (mean \pm SE) of saturated fatty acids $(\mathrm{g} / 100 \mathrm{~g}$ FA) in the milk of Chinampo cows fed alfalfa hay (HA) in barn, or freerange grazing on green forage $(\mathrm{FV})$.

\begin{tabular}{crc}
\hline \multirow{2}{*}{ Ácido graso } & \multicolumn{2}{c}{ Tratamiento } \\
\cline { 2 - 3 } & \multicolumn{1}{c}{ HA } & FV \\
\hline $14: 0$ & $11,76 \pm 0,61$ & $9,12 \pm 0,55^{*}$ \\
$15: 0$ & $2,50 \pm 0,39$ & $0,94 \pm 0,13^{* *}$ \\
$16: 0$ & $41,57 \pm 1,50$ & $31,28 \pm 1,04^{* *}$ \\
$17: 0$ & $0,65 \pm 0,30$ & $0,87 \pm 0,12$ \\
$18: 0$ & $6,57 \pm 0,53$ & $11,26 \pm 0,72^{* *}$ \\
$20: 0$ & $0,20 \pm 0,01$ & $0,39 \pm 0,06$ \\
$22: 0$ & $0,17 \pm 0,01$ & $0,28 \pm 0,05$ \\
\hline Total & $63,95 \pm 1,54$ & $54,48 \pm 1,44^{*}$ \\
\hline
\end{tabular}

Valores con asteriscos indican diferencias significativas en ácidos grasos en la misma fila: * $(\mathrm{P}<0,05) ; * *(\mathrm{P}<0,01)$. 
Cuadro 2. Concentración (media $\pm \mathrm{EE}$ ) de ácidos grasos monoinsaturados ( $\mathrm{g} / 100 \mathrm{~g}$ de $\mathrm{AG}$ ) en la leche de vacas Chinampas alimentadas con heno de alfalfa (HA) en confinamiento o con forraje verde $(\mathrm{FV})$ en pastoreo.

Concentration (mean $\pm \mathrm{SE}$ ) of monounsaturated fatty acids (g/100g FA) in the milk of Chinampo cows fed alfalfa hay (HA) in barn, or free-range grazing on green forage $(\mathrm{FV})$.

\begin{tabular}{lcc}
\hline \multirow{2}{*}{ Ácido graso } & \multicolumn{2}{c}{ Tratamiento } \\
\cline { 2 - 3 } & HA & FV \\
\hline $14: 1 n-5$ & $1,62 \pm 0,19$ & $0,71 \pm 0,04^{* *}$ \\
$15: 1 n-8$ & $0,32 \pm 0,11$ & $0,43 \pm 0,03$ \\
$16: 1 n-9$ & $0,11 \pm 0,03$ & $0,28 \pm 0,05$ \\
$16: 1 n-7$ & $3,00 \pm 0,49$ & $1,45 \pm 0,20^{* *}$ \\
$16: 1 n-5$ & $0,44 \pm 0,18$ & $0,69 \pm 0,12$ \\
$17: 1 n-7$ & $0,88 \pm 0,06$ & $0,45 \pm 0,05^{* *}$ \\
$18: 1 n-9$ & $19,81 \pm 1,29$ & $27,27 \pm 1,65^{*}$ \\
$18: 1 n-7$ & $0,85 \pm 0,06$ & $0,94 \pm 0,05$ \\
$18: 1 n-5$ & $0,34 \pm 0,07$ & $0,32 \pm 0,03$ \\
$20: 1 n-11$ & $0,10 \pm 0,03$ & $0,41 \pm 0,07^{*}$ \\
$20: 1 n-9$ & $0,14 \pm 0,02$ & $0,21 \pm 0,07$ \\
$20: 1 n-7$ & $0,06 \pm 0,01$ & $0,47 \pm 0,08^{*}$ \\
$22: 1 n-11$ & $0,11 \pm 0,036$ & $0,23 \pm 0,05$ \\
$24: 1 n-9$ & $0,06 \pm 0,01$ & $0,56 \pm 0,08^{* *}$ \\
\hline Total monoinsaturados & $29,43 \pm 1,79$ & $36,79 \pm 1,59^{*}$ \\
\hline
\end{tabular}

Literales diferentes en las hileras indican desigualdad: * $(\mathrm{P}<0,05)$; $* *(\mathrm{P}<0,01)$.

De los MUFA de cadena larga, el más abundante fue 18:1 n-9, representando más del 19,8 y 27,3 g por 100 de AG de la leche en HA y FV, respectivamente.

\section{ÁCIDOS GRASOS POLIINSATURADOS (PUFA)}

En el cuadro 3 se observa que la concentración total de PUFA fue similar en la leche de vacas alimentadas con HA y FV (P > 0,05). Con excepción del 18:2 n-6, que fue significativamente más abundante cuando las vacas se alimentaron con FV $(\mathrm{P}<0,05)$. La mayor parte de los otros PUFA no fueron significativamente diferentes.

Desde el punto de vista de la posición de un doble enlace en la cadena carbonada a partir del grupo metilo terminal, los AG omega-6 fueron más abundantes en el tratamiento FV que en HA $(\mathrm{P}<0,01)$ y los AG omega-3 presentaron diferencia no significativa $(\mathrm{P}>0,05)$.

\section{ÁCIDOS LINOLEICOS CONJUGADOS (CLA) Y MUFA TRANS}

En el cuadro 4 se observa que la concentración de 18:2 $n-6$ trans y de 18:2 n-7 cis-9 trans-11 fueron mayores en leche de vacas alimentadas con FV $(\mathrm{P}<0,05)$. Esto contribuyó a que el total de CLA resultara superior $(\mathrm{P}<0,05)$ en la leche de vacas alimentadas con forraje verde, aun si no hubo diferencia para el 18:2 n-6 trans-10 cis-12. Por otro lado, 18:1 n-7 trans-11 también fue mayor en leche de vacas alimentadas con $\mathrm{FV}(\mathrm{P}<0,05)$.

Cuadro 3. Concentración (media $\pm \mathrm{EE}$ ) de ácidos grasos poliinsaturados y altamente poliinsaturados ( $\mathrm{g} / 100 \mathrm{~g}$ de $\mathrm{AG}$ ) en la leche de vacas Chinampas alimentadas con heno de alfalfa (HA) en confinamiento o con forraje $(\mathrm{FV})$ verde en pastoreo.

Concentration (mean $\pm \mathrm{SE}$ ) of polyunsaturated fatty acids (g/100g FA) in the milk of Chinampo cows fed alfalfa hay (HA) in barn, or free-range grazing on green forage (FV).

\begin{tabular}{|c|c|c|}
\hline \multirow{2}{*}{ Ácido graso } & \multicolumn{2}{|c|}{ Tratamiento } \\
\hline & HA & $\mathrm{FV}$ \\
\hline \multicolumn{3}{|l|}{ Poliinsaturados (PUFA) } \\
\hline $18: 2 n-6$ & $1,47 \pm 0,22$ & $3,01 \pm 0,16^{* *}$ \\
\hline $18: 3 n-6$ & $0,09 \pm 0,01$ & $0,32 \pm 0,06$ \\
\hline $18: 3 n-3$ & $1,29 \pm 0,07$ & $1,06 \pm 0,14$ \\
\hline $20: 2 n-6$ & $0,09 \pm 0,01$ & $0,13 \pm 0,01$ \\
\hline $20: 3 n-6$ & $0,19 \pm 0,03$ & $0,31 \pm 0,03$ \\
\hline Total PUFA & $6,03 \pm 0,82$ & $7,91 \pm 0,85$ \\
\hline \multicolumn{3}{|c|}{ Altamente poliinsaturados (HUFA) } \\
\hline $20: 4 n-6$ & $0,17 \pm 0,04$ & $0,25 \pm 0,05$ \\
\hline $20: 5 n-3$ & $0,22 \pm 0,03$ & $0,19 \pm 0,02$ \\
\hline $22: 6 n-3$ & $1,65 \pm 0,31$ & $0,79 \pm 0,20$ \\
\hline Total HUFA & $2,04 \pm 0,38$ & $1,23 \pm 0,27$ \\
\hline Omega-3 & $3,16 \pm 0,41$ & $2,04 \pm 0,36$ \\
\hline Omega-6 & $2,37 \pm 0,37$ & $5,1 \pm 0,44 * *$ \\
\hline Relación omega-6:omega-3 & 0,75 & 2,5 \\
\hline
\end{tabular}

Literales diferentes en las hileras indican desigualdad: $* *(\mathrm{P}<0,01)$.

Cuadro 4. Concentración (media $\pm \mathrm{EE}$ ) de ácidos grasos trans (g/100 g de AG) en la leche de vacas Chinampas alimentadas con heno de alfalfa (HA) en confinamiento o con forraje (FV) verde en pastoreo.

Concentration (mean $\pm \mathrm{SE}$ ) of trans fatty acids $(\mathrm{g} / 100 \mathrm{~g}$ FA) in the milk of Chinampo cows fed alfalfa hay (HA) in barn, or free-range grazing on green forage (FV).

\begin{tabular}{lcc}
\hline & \multicolumn{2}{c}{ Tratamiento } \\
\hline Ácido graso & HA & FV \\
\hline $18: 1 n$-9 trans & $0,42 \pm 0,09$ & $0,58 \pm 0,04$ \\
$18: 1 n-7$ trans -11 & $0,98 \pm 0,20$ & $1,57 \pm 0,14^{*}$ \\
$18: 2 n-6$ trans & $0,30 \pm 0,05$ & $0,91 \pm 0,10^{* *}$ \\
$18: 2 n-7$ cis- 9 trans -11 & $0,50 \pm 0,04$ & $0,77 \pm 0,05^{*}$ \\
$18: 2 n-6$ trans -10 cis- 12 & $0,06 \pm 0,01$ & $0,17 \pm 0,03$ \\
\hline Total trans & 2,26 & 4,0 \\
\hline $18: 2 n-7$ cis- 9 trans -11 & 1,48 & 2,34 \\
$+18: 1$ n-7 trans -11 & & \\
\hline
\end{tabular}

Literales diferentes en las hileras indican desigualdad: * $(\mathrm{P}<0,05)$; ** $(\mathrm{P}<0,01)$. 


\section{DISCUSIÓN}

La concentración total de lípidos en ambos tratamientos está debajo del rango reportado para vacas Holstein alimentadas con forraje verde en pastoreo (Frelich y col 2009) y para vacas de doble propósito en pastoreo sobre forraje verde y suplementadas con diferentes cantidades de materia seca (Rico y col 2007).

La concentración de AG saturados en la leche de las vacas alimentadas con heno de alfalfa está dentro del rango reportado en vacas lecheras. Sin embargo, en los animales que se alimentaron con forraje verde, dicho valor se encuentra por debajo del mínimo señalado por Månsson (2008). Este autor menciona que el contenido de AG saturados en la leche fue menor durante el verano, etapa en que las vacas se mantienen en pastoreo, comparado con el invierno, cuando los animales permanecen estabulados y consumen alimento seco.

En otros experimentos, Galina y col (2007) midieron las concentraciones de $\mathrm{AG}$ en quesos elaborados con leche de vacas Cebú y de cabras que pastorearon en pradera de una leguminosa del bosque tropical, o estabuladas y alimentadas con ensilaje de maíz más un suplemento basado en concentrado. En esos estudios el consumo de forraje verde redujo la concentración de AG saturados del queso en ambas especies. En nuestro estudio, los AG saturados más abundantes fueron 16:0 y 14:0, con concentraciones mayores a las reportadas en vacas lecheras de la sabana colombiana (Rico y col 2007). Proporciones similares de 14:0 e inferiores de 16:0 en el grupo FV del presente trabajo fueron determinadas en la leche de vacas Holstein pastoreando sobre alfalfa en Argentina (Castillo y col 2006).

El total de MUFA resultó mayor en las vacas del tratamiento FV. Esto coincide con los resultados de otro estudio en el que se comparó la misma variable en la leche de vacas pastoreando sobre forraje verde o alimentadas con ensilaje (Frelich y col 2009). Los MUFA de cadena mediana en el grupo HA se encontraron dentro del rango reportado en otros estudios. Sin embargo, los valores registrados en las vacas alimentadas con FV están por debajo de los referidos para vacas lecheras (Jensen 2002).

En nuestro trabajo se observó una mayor cantidad de 16:1 n-7 en HA. Aunque no se cuantificaron los AG del forraje, esto quizá se relaciona con una mayor concentración del AG referido en el heno de alfalfa, como ocurrió en un estudio con vacas Holstein (Dhiman y col 1999).

Aunque la concentración de PUFA fue similar entre $\mathrm{HA}$ y FV, la tendencia favorece al segundo grupo. En este sentido, nuestros resultados coinciden con los de Frelich y col (2009). Lo anterior coincide también con los resultados de un estudio realizado con vacas Simmental bajo pastoreo de forraje verde, en un sistema de producción orgánica (Vranješ y col 2010).

De los PUFA, solamente el 18:2 $n$-6 y el 18:2 $n$-7 cis-9 trans-11 fueron afectados por la dieta. Ambos aumentaron en la leche de vacas alimentadas con forraje verde. Las concentraciones del 18:2 n-7 cis-9 trans-11 en HA y FV están dentro del rango señalado en la literatura (Kelly y col 1998). La mayor concentración del 18:2 n-6 en la leche del grupo FV podría estar asociada con un mayor consumo del AG en cuestión, o con una menor biohidrogenación debido a la actividad de la polifenol oxidasa y de los fenoles (Steinshamn y col 2007). Estos compuestos se encuentran en altas concentraciones en los arbustos que forman parte de la dieta del ganado que pastorea en el área donde se realizó el estudio (Ramírez-Orduña y col 2003). La composición lipídica de los forrajes verdes consiste en glucolípidos y fosfolípidos, con una concentración alta de 18:2 n-6 y 18:3 n-3, principalmente. Estos AG son sustratos importantes para la biohidrogenación por los microorganismos del rumen (Jenkins y col 2008). A partir de los ácidos 18:2 n-6 y 18:3n-3, se producen los isómeros de valor biológico como los ácidos 18:2 n-7 cis-9 trans-11 y 18:1 $n-7$ trans-11 (Jalc y col 2007). Es importante mencionar que el ácido 18:3 n-3 no sintetiza 18:2 $n-7$ cis-9 trans- 11 en el metabolismo ruminal, pero resulta en la formación de 18:1 n-7 trans-11 como el principal intermediario (Harfoot y Hazlewood 1988), importante en la fuente de 18:2 n-7 cis-9 trans-11 en la grasa de la leche a nivel de glándula mamaria (Griinari y Bauman 1999).

Los ácidos 18:2 $n-7$ cis-9 trans-11 y 18:1 $n-7$ trans- 11 que pasan del rumen al intestino delgado son absorbidos y transportados a la glándula mamaria; aquí, el ácido 18:1 n-7 trans-11 es desaturado a ácido 18:2 n-7 cis-9 trans-11 y este último se libera en la leche (Bauman y col 2006). Esto explica por qué el contenido de CLA en la grasa de la leche de vacas alimentadas en pastoreo es mayor que en las vacas que comieron una ración integral (Schroeder y col 2003, Stockdale y col 2003, Frelich y col 2009).

Con base en esto se puede argumentar que los resultados observados en el presente estudio se pudieran relacionar con una cantidad menor de ácido 18:3n-3 presente en el heno de alfalfa, ya que, por pérdidas oxidativas durante la henificación, dicho AG se reduce hasta una sexta parte de lo que contiene el zacate fresco (Tanaka 2005). Esas pérdidas están asociadas con el sistema lipoxigenasa, un mecanismo de defensa de las plantas ante el daño tisular que termina catabolizando los PUFA a compuestos volátiles como aldehídos y alcoholes (Dewhurst y col 2006).

De los resultados del presente estudio se concluye que la alimentación de vacas Chinampas con forraje verde en pastoreo modificó la composición de la grasa de la leche, redujo el contenido de ácidos grasos saturados de cadena mediana e incrementó la proporción de MUFA, entre ellos el 18:1 n-7 trans-11. Además, aumentó la cantidad del CLA 18:2 n-7 cis-9 trans-11, de gran importancia desde el punto de vista de la salud humana. Así, el pastoreo en forrajes verdes modifica la composición de la grasa de la leche haciéndola más benéfica para la salud de la población humana que consume el producto. 
La composición específica de la grasa de la leche producida por vacas en pastoreo merece también la atención de los procesadores de leche y puede ser evaluada positivamente por los consumidores. Los resultados implican también que el consumo humano del 18:2 n-7 cis-9 trans-11 se pueda incrementar aumentando la ingestión de leche de vaca, o ampliando el contenido de 18:2 n-7 cis-9 trans-11 en los productos lácteos derivados del bovino. La segunda alternativa resulta más práctica. Sin embargo, aun si a nivel de salud humana una menor concentración de AG saturados es un buen resultado y la cantidad de ácido 18:2n-7 cis-9 trans-11 y 18:1 n-7 trans-11 aumenta, sería deseable que la leche presentara menores niveles en la relación omega-6:omega-3.

\section{RESUMEN}

El objetivo del estudio fue determinar la concentración de ácidos grasos (AG) en la leche de vacas Chinampas, con énfasis en los ácidos grasos conjugados (CLA). Se colectó una muestra manual de leche (10 ml) de 32 vacas entre los 60 y 120 días de lactación. Un grupo (FV; $\mathrm{n}=25$ ) se alimentó en pastoreo con forraje verde durante la estación de lluvias de septiembre a octubre de 2009 en una zona semiárida de matorral sarcocaulescente (Municipio La Paz, B.C.S.). Otro grupo (HA; $n=7)$ se mantuvo en corral y se alimentó con heno de alfalfa. El análisis cromatográfico revela que la concentración de AG saturados fue menor $(\mathrm{P}<0,05)$ en FV. Esa diferencia estuvo determinada por los AG 14:0, 15:0 y 16:0, principalmente. El total de AG monoinsaturados fue mayor en FV $(\mathrm{P}<0,05)$, debido al incremento de 18:1 n-9 y 18:1 n-7 trans-11. El total de AG poliinsaturados fue similar entre grupos, pero los AG 18:2 n-6 y 18:2 n-7 cis-9 trans-11 fueron más abundantes en FV $(\mathrm{P}<0,01)$. La proporción de AG omega-3 fue parecida entre tratamientos y la concentración de AG omega- 6 fue mayor en FV ( $\mathrm{P}<0,01)$, por lo que la relación de omega-6:omega-3 fue mayor en FV. Se concluye que la alimentación de vacas Chinampas con forraje verde en pastoreo redujo el contenido de AG saturados de cadena mediana e incrementó la concentración de los AG monoinsaturados 18:1 n-9, del 18:1 n-7 trans-11 y del ácido conjugado 18:2 n-7 cis-9 trans-11, siendo así benéfico para la salud humana. Sin embargo, sería deseable incrementar los niveles de omega-3 en la leche de vacas alimentadas con FV.

\section{REFERENCIAS}

Ávalos CR, RMG Chávez, CJC Leyva, AJD Osuna, HM Sánchez. 2009. Valor nutritivo y consumo voluntario del forraje disponible para ganado Criollo en dos estaciones del año en la baja sur, México. XIX Reunión Internacional sobre Producción de Carne y Leche en Climas Cálidos, Mexicali, BC, México, Pp 69-73.

Bauman DE, IH Mather, RJ Wall, AL Lock. 2006. Major advances associated with the biosynthesis of milk. J Dairy Sci 89, 1235-1243.

Bligh GE, JW Dyer. 1959. A rapid method of total lipid extraction and purification. Can J Biochem Physiol 37, 911-917.

Castillo A, MA Taverna, R Páez, A Cuatrin, D Colombatto, F Bargo, M García, P García, M Chávez, A Beaulieu, J Drackley. 2006. Fatty acids composition of milk from dairy cows fed fresh alfalfa based diets. Anim Feed Sci Tech 131, 241-254.

Chand A, A Wilkins, E Kolver, M Veth. 2001. A rapid method for quantification of conjugated linoleic acid in rumen fluid using gas chromatography. Analytical Science. Supplement The Japan Society for Analytic Chemistry 17, a397-a400.

Chilliard Y, A Ferlay. 2004. Dietary lipids and forages interactions on cow and goat milk fatty acid composition and sensory properties. Reprod Nutr Dev 44, 467-492.
Clandinin MT, SL Cook, SD Konrad, MA French. 2000. The effect of palmitic acid on lipoprotein colesterol levels. Int J Food Sci Nutr 51, S61-S71.

Collomb M, R Sieber, U Bütikofer. 2004. CLA isomers in milk fat from cows fed diets with high levels of unsaturated fatty acids. Lipids 39, 355-364.

Coppa M, A Ferlay, F Monsallier, I Verdier-Metz, P Pradel, R Didienne, A Farruggia, MC Montel, B Martin. 2011. Milk fatty acid composition and cheese texture and appearance from cows fed hay or different grazing systems on upland pastures. J Dairy Sci 94, 1132-1145.

Cruz-Hernandez C, Z Deng, J Zhou, AR Hill, MPYurawecz, P Delmonte, MM Mossoba, ME Dugan, JK Kramer. 2004. Methods for analysis of conjugated linoleic acids and trans-18:1 isomers in dairy fats by using a combination of gas chromatography, silver-ion thinlayer chromatography/gas chromatography, and silver-ion liquid chromatography. J AOAC Int 87, 545-562.

Destaillats F, RL Wolfe, D Precht, J Molkentin. 2000. Study of individual trans and cis-16:1 isomers in cow, goat, and ewe cheese fats by gas-liquid chromatography with emphasis on the trans-3 isomer. Lipids 35, 1027-1032.

Dewhurst RJ, KJ Shingfield, MRF Lee, ND Scollan. 2006. Increasing the concentrations of beneficial polyunsatured fatty acids in milk produced by dairy cows in high-forage systems. Anim Feed Sci Tech 131, 168-206.

Dhiman TR, GR Anand, LD Satter, MW Pariza. 1999. Conjugated linoleic acid content of milk from cows fed different diets. J Dairy Sci 82, 2146-2156.

Duckett SK, JPS Neel, JP Fontenot, WM Clapham. 2009. Effects of winter stocker growth rate and finishing system on: III. Tissue proximate, fatty acid, vitamin, and cholesterol content. J Anim Sci 87, 2961-2970

Espinoza JL, J Sánchez, JA Gracia, JR Sánchez, R Ortega, A Palacios. 2009. Thermoregulation differs in Chinampo (Bos taurus) and locally born dairy cattle. Turk J Vet Anim Sci 33, 175-180.

Ferlay A, C Agabriel, C Sibra, C Journal, B Martin, Y Chilliard. 2008. Tanker milk variability in fatty acids according to farm feeding and husbandry practices in a French semi-mountain area. Dairy Sci Tech 88, 193-215.

Field CJ, HH Blewett, S Proctor, D Vine. 2009. Human health benefits of vaccenic acid. Appl Physiol Nutr Metab 34, 979-991.

Frelich J, M Šlachta, O Hanuš, J Špička, E Samková. 2009. Fatty acid composition of cow milk fat produced on low-input mountain farms. Czech J Anim Sci 54, 532-539.

Galina MA, F Osnaya, HM Cuchillo, GFW Haenlein. 2007. Cheese quality from milk of grazing or indoor fed Zebu cows and Alpine crossbred goats. Small Rumin Res 71, 264-272.

Griinari JM, DE Bauman. 1999. Biosynthesis of conjugated linoleic acid and its incorporation into meat and milk in ruminants. In: Yurawcez MP, Mossoba MM, Kramer JKG, Pariza MW, Nelson GJ (eds). Advances in conjugated linoleic acid research. Vol. 1. AOCS Press, Illinois, USA, Pp 180-200

Harfoot CG, GP Hazlewood. 1988. Lipid metabolism in the rumen. In Hobson PN (ed). The rumen microbial ecosystem. Elsevier Science, London, UK, Pp 285-322.

Izumi Y, JK An, Y Kobayashi, K Tanaka. 2002. Effects of fresh grass feeding on the formation of conjugated linoleic acid (CLA) and vaccenic acid (trans-11C18:1) in the rumen. Proceedings of Japanese Society for Rumen Metabolism and Physiology 15, 43-46.

Jacobs MN, A Covaci, A Gherghe, P Schepen. 2004. Time trend investigation of PCBs, PBDEs and organochlorine pesticides in selected $n-3$ polyunsaturated fatty acid rich dietary fish oil and vegetable oil supplements; nutritional relevance for human essential $n-3$ fatty acid requirements. J Agric Food Chem 52, 1780-1788.

Jalc D, M Certik, K Kundrikova, P Namestkova. 2007. Effect of unsaturated $\mathrm{C} 18$ fatty acids (oleic, linoleic and $\alpha$-linolenic acid) on ruminal fermentation and production of fatty acid isomers in an artificial rumen. Vet Med 52, 87-94. 
Jenkins TC, RJ Wallace, PJ Moate, EE Mosley. 2008. Recent advances in biohydrogenation of unsaturated fatty acids within the rumen microbial ecosystem. J Anim Sci 86, 397-412.

Jensen RG. 2002. The composition of bovine milk lipids. J Dairy Sci 85, 295-350.

Kelly ML, JR Berry, DA Dwyer, JM Griinari, PY Chouinard, MEV Amburgh, DE Bauman. 1998. Dietary fatty acid sources affect conjugated linoleic acid concentrations in milk from lactating dairy cows. J Nutr 128, 881-885.

Kelsey JA, BA Corl, RJ Collier, DE Bauman. 2003. The effect of breed, parity and stage of lactation on conjugated linoleic acid (CLA) in milk fat from dairy cows. J Dairy Sci 86, 2588-2597.

Khanal RC, KC Olson. 2004. Factors affecting conjugated linoleic acid (CLA) content in milk, meat, and egg: A Review Pakistan J Nutr 3, 82-98.

Kramer JKG, PW Parodi, RG Jensen, MM Mossoba, MP Yurawecz, RO Adlof. 1998. Rumenic acid: A proposal common name for the major conjugated linoleic acid isomer found in natural products. Lipids 33, 853 (Abstr.).

Lawless F, JJ Murphy, D Harrington, R Devery, C Stanton. 1999. Elevation of conjugated cis-9,trans-11- octadecadienoic acid in bovine milk because of dietary supplementation. J Dairy Sci 81, 3259-3267.

Leheska JM, LD Thompson, JC Howe, E Hentges, J Boyce, JC Brooks, B Shriver, L Hoover, MF Miller. 2008. Effects of conventional and grass-feeding systems on the nutrient composition of beef. J Anim Sci 86, 3575-3585.

Lock AL, DE Bauman. 2004. Modifying milk fat composition of dairy cows to enhance fatty acids beneficial to human health. Lipids 39, 1197-1206.

Lock AL, KJ Shingfield. 2004. Optimising milk composition. In: Kebreab E, Mills J, Beever DE (eds). Dairying-Using Science to Meet Consumers'Needs. Occ. Pub. $N^{\circ} 29$ Brit Soc Anim Sci, Nottingham University Press, Loughborough, UK, Pp 107-188.

Månsson HL. 2008. Fatty acids in bovine milk fat. Food Nutr Res DOI: 10.3402/fnr.v52i0.1821.

Martin SA, TC Jenkins. 2002. Factors affecting conjugated linoleic acid and trans- $\mathrm{C}_{18: 1}$ fatty acid production by mixed ruminal bacteria. $J$ Anim Sci 80, 3347-3352.

Morelos OS. 1988. La vegetación: una aproximación a través de la fotointerpretación en la Sierra de La Laguna de Baja California
Sur. Centro de Investigaciones Biológicas de B.C.S. A.C. La Paz, B. C. S., México.

Moya-Camarena SY, JP Vanden Heuvel, SG Blanchard, LA Leesnitzer, MA Belury. 1999. Conjugated linoleic acid is a potent naturally occurring ligand and activator of PPAR $\alpha$. Lipid Res 40, 1426-1433.

Ramírez-Orduña R, RG Ramírez, MV Gómez-Meza, JA ArmentaQuintana, JM Ramírez-Orduña, R Cepeda-Palacios, JM Ávila-Sandoval. 2003. Seasonal dynamics of organic matter digestion in browse species from Baja California Sur, Mexico. $J$ Appl Anim Res 24, 65-78.

Rico JE, B Moreno, ML Pabón, J Carulla. 2007. Composición de la grasa láctea en la sabana de Bogotá con énfasis en ácido ruménico - CLA cis-9, trans-11. Rev Col Cienc Pec 20, 30-39.

SAS. 2001. SAS User'S Guide (Release 8.2). Statistics SAS Inst. Inc., Cary. North Caroline, USA.

Schroeder GF, JE Delahoy, I Vidaurreta, F Bargo, JA Gagliostro, LM Muller. 2003. Milk fatty acid composition of cows fed a total mixed ration or pasture plus concentrates replacing corn with fat. J Dairy Sci 86, 3237-3248.

Simopoulos A. 2008. The importance of the omega-6/omega-3 fatty acid ratio in cardiovascular disease and other chronic disease. Exp Biol Med 233, 674-688.

Steinshamn H, E Thuen, UT Brenøe. 2007. Clover species in grass-clover silages affects milk fatty acid composition. J Anim Feed Sci 16, 65-69.

Stockdale CR, GP Walker, WJ Wales, DE Dalley, A Birkett, Z Shen, PT Doyle. 2003. Influence of pasture and concentrates in the diet of grazing dairy cows on the fatty acid composition of milk. J Dairy Sci 70, 267-276.

Tanaka K. 2005. Occurrence of conjugated linoleic acid in ruminant products and its physiological functions. Anim Sci J 76, 291-303.

Valsta LM, H Tapanainen, S Männistö. 2005. Meat fats in nutrition: a review. Meat Sci 70, 525-530.

Vranješ AP, M Krajinović, J Kecman, S Trivunović, R Pejanović, G Krajinović, G Mačak. 2010. Comparison of fatty acid composition in conventional and organic milk. Mljekarstvo 60, 59-66.

Whitman RH. 1975. Weight changes, body condition and beef-cow reproduction. PhD Dissertation, Colorado State University, Fort Collins, CO, USA.

Williams CM. 2000. Dietary fatty acids and human health. Ann Zootech $49,165-180$. 